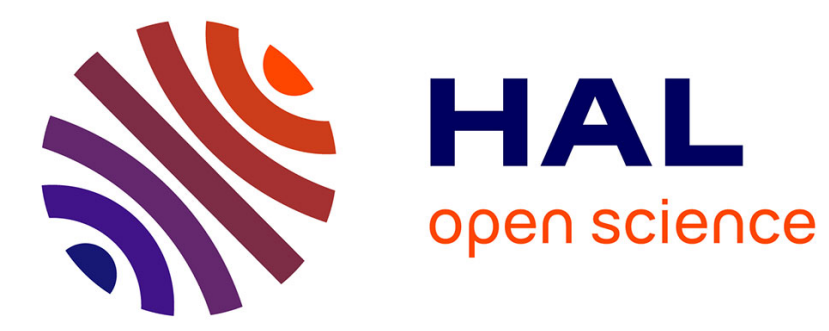

\title{
The collapse of insensitive semantics
}

\author{
Friedrich Christoph Doerge
}

\section{To cite this version:}

Friedrich Christoph Doerge. The collapse of insensitive semantics. Linguistics and Philosophy, 2010, 33 (2), pp.117-140. 10.1007/s10988-010-9077-y . hal-00643843

\section{HAL Id: hal-00643843 \\ https://hal.science/hal-00643843}

Submitted on 23 Nov 2011

HAL is a multi-disciplinary open access archive for the deposit and dissemination of scientific research documents, whether they are published or not. The documents may come from teaching and research institutions in France or abroad, or from public or private research centers.
L'archive ouverte pluridisciplinaire HAL, est destinée au dépôt et à la diffusion de documents scientifiques de niveau recherche, publiés ou non, émanant des établissements d'enseignement et de recherche français ou étrangers, des laboratoires publics ou privés. 


\section{Abstract:}

The idea motivating their account, Cappelen and Lepore say in Insensitive Semantics (2005), is that semantic content is context invariant, and that all colleagues who take, or even consider, different accounts are just on the wrong track. It is the purpose of their book to disprove all alternative accounts by way of an argument 'by elimination'. The conclusion they arrive at is that their own account must be accepted by everyone as "the only game in town at the end of the day". The present paper is intended to examine this conclusion; its more significant findings are these. Firstly, C\&L's account is not, as they suggest, strictly minimalist, but in fact just a moderate version of contextualism. Secondly, prematurely associating semantical incompleteness and context sensitivity, they overlook some possible alternatives to their own view, among them at least one that is attractive. Thirdly, their argument 'by elimination' has an inductive structure, but is inexhaustive, and therefore inconclusive. Fourthly, for several different reasons, their attempts to reject arguments in favour of semantical incompleteness do not work. Finally, their contention that arguments in favour of semantical incompleteness employ metaphysical premisses for semantical conclusions rests on a faulty interpretation of these arguments. In the light of these findings, it is concluded that the central argument of Insensitive Semantics fails.

\section{Introduction ${ }^{1}$}

“The idea motivating Semantic Minimalism", Cappelen and Lepore (C\&L) say in Insensitive Semantics (2005, 143), "is simple and obvious: The semantic content ${ }^{2}$ of a sentence $S$ is the content that all utterances of S share"; it is "the content that can be grasped and reported by someone who is ignorant about the relevant characteristics of the context in which an utterance of S took place". ${ }^{3}$ I emphatically agree both to the truth and the significance of this view; following C\&L themselves (2005, 143), I shall call it the "Basic Idea" of their account.

According to the Basic Idea, there is no contribution of context to semantic content at all. What C\&L subsequently defend as "Semantic Minimalism", however, is something slightly different: it is the view that there is no contribution of the context to semantic meaning with the exception of members of the set 
of (genuinely) context sensitive expressions $(2005,17,151)$. I shall call this the "Basic Set"; its members amount, at least roughly, to those expressions which Kaplan (1989) calls "demonstratives" (cf. C\&L 2005, 1f.). Because of the exception they make, calling their view "Semantic Minimalism", as they do themselves, is a bit misleading; in the present paper I shall instead call it "Very Moderate Contextualism" (VMC). A view for which the term 'minimalist' is more appropriate will be introduced below. But let me first continue presenting C\&L's case.

The central task of Insensitive Semantics $(I S)$ is to show that VMC must be true, because all alternative views are unacceptable. In order to achieve it, C\&L present what they $(2005,151)$ call an argument "by elimination". The argument starts from the assumption that there are exactly two alternatives to VMC; one is what they call "Moderate Contextualism", the other is called "Radical Contextualism" (C\&L 2005, 151, 190). Moderate Contextualism (MC) is the view that, besides demonstratives, there are other expressions which are semantically incomplete and context sensitive (2005, 7, 39, 59). Radical Contextualism (RC) says that each and every sentence, ${ }^{4}$ or even each and every expression, ${ }^{5}$ is context sensitive, and semantically incomplete. ${ }^{6}$ So in the main, ${ }^{7}$ C\&L construe the difference between MC and RC as a difference concerning the extension of context sensitivity and semantical incompleteness (we turn back to this issue presently).

Now as to the argument by elimination, very roughly, it works as follows. Firstly, in C\&L's view RC is a completely unacceptable view. In order to convince us of this, they raise three objections against RC. Firstly, they argue, RC fails the so-called "context sensitivity test", thus assuming context sensitivity in cases where, on their own view, there is none. ${ }^{8}$ Secondly, on their view RC fails to explain the pervasive presence of communicative success; indeed, they argue, if $\mathrm{RC}$ was true, then communication would be impossible. ${ }^{9}$ Thirdly, they argue that RC is an inconsistent view, because assuming it leads to contradictions. ${ }^{10}$ Thus, they $(2005,14,151)$ arrive at a devastating assessment of RC: it is "internally inconsistent" and "empirically flawed", and it "fails to account for some of the basic features of our linguistic practices". 
As to MC, according to C\&L it is in no better a position. For although it appears to constitute a more moderate position at first, C\&L argue, it finally turns out to force its adherent to the endorsement of RC. C\&L construe what they call a "Slippery Slide Argument" (SSA), an argument which sets out to show that all attempts to establish MC entail a commitment to the endorsement of RC, such that after all MC cannot be defended without simultaneously endorsing RC. More in particular, the argument proceeds as follows.

"[A]t the center of all arguments that attempt to establish" MC, they $(2005,17)$ argue, are two and only two arguments: "Context Shifting Arguments" and "Incompleteness Arguments". ${ }^{11}$ Context Shifting Arguments bring to bear intuitions which suggest that "what is said, or expressed by, or the truth conditions of, an utterance varies in some systematic way with contexts of utterance" $(2005,10)$, thus suggesting that the sentence under consideration is context-sensitive. Incompleteness Arguments, again, employ intuitions to the effect that a certain sentence, or expression, is semantically incomplete (these will be considered in more detail below). What the SSA is designed to show is this: Regardless whether adherents of MC employ an Incompletess Argument, or a Context Shifting Argument, in either case do they "lead themselves directly into the clutches of RC" $(2005,7)$. Thus, whatever argument is employed in favour of MC, to produce it means to endorse RC.

Now given this commitment of arguments in favour of MC to an endorsement of RC, C\&L appear to have established their case. MC and RC are the only alternatives to VMC. MC collapses into RC, and thus, they conclude, "RC [...] is the logical consequence of denying" VMC $(2005,7)$. Furthermore, RC appears to be completely unacceptable. Thus, there appears to be no alternative to endorsing VMC. As C\&L put it themselves: apparently, VMC remains as "the only game in town at the end of the day" (2005, 151; cf. also 190).

In the few years since the publication of their book, a profound number of replies have been given. Indeed, though it sounds appealing at first, most of their opponents remain decidedly reluctant to subscribe to their account and to swallow the consequences it brings with it, after all. Still, the central argument of $I S$, by which they set out to show that all other views are unacceptable, has not yet been 
scrutinised conclusively up to now. The present paper is intended to contribute to this task. It shows that, in several different ways, C\&L's argument fails to work.

As a start, in the remainder of this section I shall abandon an assumption which is presupposed by C\&L's argument, but which appears to be unfounded, namely, the strict association of context sensitivity and semantical incompleteness with each other. This view underlies the simple trichotomy which C\&L construe, consisting of $\mathrm{RC}, \mathrm{MC}$, and $\mathrm{VMC}$. For the claim that these three views exhaust the range of possible accounts makes sense only on the assumption that context sensitivity and semantical incompleteness strictly cooccur. This assumption, however, is highly doubtful.

To start with, it is at least not the case that the two terms, "context sensitivity" and "semantical incompleteness" are just different words for one and the same thing. Nor is this entailed, or even suggested, by the way in which C\&L's describe these terms. According to C\&L (2005, 33), context sensitivity is "variability between contexts", and "to establish such variability one has to think about and compare what happens to the same sentence in different contexts of utterance"; a sentence is context sensitive when its semantic content "shift[s] from context to context" (2005, 105; cf. 2005, 33, 99, 104).

The notion of semantical incompleteness is described quite differently. "In the case of incompleteness", the sentence under consideration "has [no] conditions of truth, and so, [no] truth value, independent of any context" $(2005,33)$. For without complementation by a context, semantically incomplete sentences express "only fragmentary propositions". Such a sentence lacks truth-conditions because "it is incomplete qua semantic entity" $(2005,7)$.

Thus, when a sentence is semantically incomplete, then this does not necessarily involve any consideration of what might happen to the sentence in one or the other context of utterance; while a sentence's context sensitivity essentially derives from its behaviour in one or the other particular context. Furthermore, when a sentence is semantically incomplete, then this is derived from the semantical incompleteness of its character, while a sentence's context sensitivity derives from the context shifting of the semantic contents associated with it in different contexts. Thus, the concept of 
context sensitivie and the concept of semantical incompleteness are different concepts, according to the description C\&L give of them.

It might still be that context sensitivity and semantical incompleteness, though different things, are coextensional. Given that the two ideas are different, however, establishing such a view would seem to require good reasons. Now, on the one hand, $C \& L$ themselves do not give any argument to this effect (they do not try to establish this view; they just do not deal with the issue). On the other hand, there are several reasons against coextensionality. Thus, it seems arguable that the expression "Napoleon Buonaparte left in the lurch" is semantically incomplete (as well as, additionally, syntactically incomplete), while not being context sensitive. On the other hand, it seems arguable that "The tallest European is bald" is context sensitive, but not semantically incomplete.

Thus, there is reason not to assume coextensionality of context sensitivity and semantical incompleteness. ${ }^{12}$ It is then a possible view, and in my opinion indeed an attractive one, that some sentences are semantically incomplete, while there is no such thing as context sensitivity-a moderate account of the extension of semantical incompleteness, that means, and a consistently 'minimal' account of the extension of context sensitivity. ${ }^{13}$ This minimal view of context sensitivity, together with a moderate endorsement of semantical incompleteness, can well be combined with different views of the nature and extension of semantic content such as, for example, with the view that not all sentences do have any semantic content in addition to a 'character' in Kaplan's (1989) sense, or with a conception of 'semantic content' according to which it is possible for a sentence to have a content which is not truthevaluable, or again, with a rather liberal view of truth-evaluability (similar, for example, to the one C\&L defend in $I S) .^{14}$

One advantage of the account I am suggesting, at least from the point of view which C\&L take, is that it takes their Basic Idea, that semantic content is not context sensitive, seriously. For this Basic Idea suggests that the semantic content of a sentence is independent of the context of utterance. By assuming no such thing as context sensitivity, the present view accounts for the Basic Idea straightforwardly- 
while by assuming a considerable amount of context sensitivity, ${ }^{15} \mathrm{C} \&$ L's own theory accounts for their Basic Idea at least less obviously.

Furthermore, there is a second advantage of my account from the point of view of C\&L's account. It is a presupposition of their central argument in $I S$ that any account of semantic content which does assume some amount of context sensitivity must defend this view somehow (while, as they argue, any such defence will cause an irresistable collapse into RC, which makes it an unacceptable view after all). Now by suggesting that after all there is some amount of context sensitivity there is reason to believe that their own account eventually succumbs to this problem; while the account I am suggesting, by assuming no context sensitivity at all, perfectly avoids problems of this type-we shall turn back to this issue below.

As far as terminology is concerned, since the account I suggest assumes no context sensitivity, I think it is appropriate to call it "Real Semantic Minimalism" (Real SM) with respect to context sensitivity. C\&L's own account of context sensitivity, however, since it finally does assume some amount of context sensitivity, may perhaps more appropriately be identified as "Very Moderate Contextualism" (VMC), rather than as "Semantic Minimalism".

Anyway, the account I suggest assumes a certain amount of semantical incompleteness, while it does not assume any amount of context sensitivity. This 'mixed' design of the view has certain significant consequences for my examination of the argument of $I S$. As we remember, C\&L's attack against competing accounts involves the rejection of two kinds of phenomena, context sensitivity and semantical incompleteness. Since I suggest taking a view according to which there is no context sensitivity, my account is immune to their arguments against context sensitivity, and thus these arguments need not be taken into consideration here.

However, my account does involve a moderate endorsement of semantical incompleteness. C\&L call arguments in favour of such an endorsement "Incompleteness Arguments". Their relevant SSA says that any Incompleteness Argument, if it is applicable at all, will be applicable to each and every sentence, 
and that the one who employs such an Incompleteness Argument thus gets committed to the endorsement of RC, which is a completely unacceptable view. After a general analysis of their overall argument in section 2, in sections 3 and 4 I shall argue that their SSA against Incompleteness Argument is unsuccessful. It is not true that any argument in favour of semantical incompleteness commits us to the view that semantical incompleteness is ubiquitous. As we shall see, there are several reasons for this conclusion. For example, their attempt to reject the two Incompleteness Arguments fails in either case, and each time in more than one way. Additionally, the two Incompleteness Arguments which they take into consideration do not exhaust the range of Incompleteness Arguments, which anyway makes the SSA inconclusive. C\&L present another objection to arguments in favour of semantical incompleteness, to the effect that such arguments employ metaphysical assumptions for the support of semantical conclusions, and are therefore unacceptable. In section 5, I examine this line of argument, and show that it is unconvincing, too.

\section{General analysis of C\&L's argument by elimination}

So C\&L set out to reject all kinds of Incompleteness Arguments: what exactly is an 'Incompleteness Argument'? Following C\&L's description, ${ }^{16}$ it is an argument that furthers the view that certain sentences are semantically incomplete. A typical instance of such an argument may, for example, sound like this.

Consider the alleged proposition that $\mathrm{P}$ that some sentence S semantically expresses. Intuitively, the world can't just be $\mathrm{P}$ simpliciter. The world is neither P nor not P. There's no such thing as P's being the case simpliciter. And so, there is no such proposition. (C\&L 2005, 11)

In general, the goal of an Incompleteness Argument, according to C\&L $(2005,11)$, "is to establish that the proposition Semantic Minimalists claim is semantically expressed by some sentence $\mathrm{S}$ is no more than a propositional fragment". That a sentence is 'semantically incomplete', means that it must be 
"completed" by "adding" something to its meaning for it to be truth-evaluable, while otherwise this meaning merely constitutes a propositional fragment (2005, 61); and that a sentence expresses a 'propositional fragment' means that it "does not express a complete proposition", but instead something that "cannot have a truth-value", and that it "doesn't express genuine truth conditions" $(2005,61)$.

Making their case against Incompletenes Arguments, C\&L advance what they call a 'Slippery Slide Argument' (SSA). In its most general form, as directed against any version of MC, and following C\&L's $(2005,39,59)$ explicit description, ${ }^{17}$ it can be represented as follows: Given an argument A, if A suffices to show that MC is true, then RC follows. To see exactly what they mean, however, some qualifications are necessary.

(1) Taken literally, this statement suggests that the SSAs applies only to arguments which "suffice to show that" MC is true. Construed this way, however, the argument would fail to rebut reasonings which aim at strong evidence for context sensitivity and semantical incompleteness, or at some evidence, rather than at conclusive evidence. Since C\&L aim at an exhaustive rejection of all arguments in favour of MC, the focus must be wider: the argument is to be construed as directed against any argument providing evidence for MC, as indeed C\&L themselves do in other places (e.g., 2005, 14). Thus, the following statement is more accurate: Given an argument A, if A provides evidence in favour of MC, then RC follows.

(2) Saying that RC "follows" from arguments in favour of MC, C\&L may appear to be claiming an $a$ priori connection between an argument's supporting MC, and its entailing RC. ${ }^{18}$ This, however, is not the case. The reason they suggest for the connection is not a priori. Nor do they claim that there is a general reason why arguments in favour of MC commit one to endorsing RC. Their SSA, rather consists of a bunch of partial SSAs, each of them directed individually against one or the other argument in favour of MC, in such a way that for each single argument in favour of MC there is at least one SSA which shows that endorsing this argument commits one to the endorsement of RC. In their own words, they execute the argument by considering particular sets of data "presented in favour of expanding the Basic Set of context sensitive [sc., and/or semantically incomplete] expressions, and show that if this 
evidence supports an expansion of the Basic Set, then all expressions are context sensitive [sc., and/or semantically incomplete], i.e., RC is true" $(2005,14)$. Thus, the SSA against MC splits up into a more or less huge number of partial SSAs, addressing each and every single argument in favour of context sensitivity and/or semantical incompleteness there is, and arguing in each case that this argument, too, commits one to the endorsement of RC.

(3) As a consequence of the inductive structure of the overall SSA, C\&L's case rests upon a variety of particular SSAs, directed against particular arguments in favour of MC. Since MC has two aspects, context sensitivity and semantical incompleteness, the entirety of arguments for MC can be split in two groups, those concerning context sensitivity, and those concerning semantical incompleteness. Since I am defending a moderate view of semantical incompleteness, I shall in the following be concerned with the SSA as directed (or those partial SSAs which are directed) against Incompleteness Arguments.

Given the fact that $\mathrm{C} \& \mathrm{~L}$ give no general reason why arguments in favour of semantical incompleteness are supposed to commit one to the endorsement of RC, and given their contention that each single Incompleteness Argument does involve such a commitment nevertheless, C\&L are forced to take each and every single Incompleteness Argument into consideration, and to try to give a reason in each case, why this particular Incompleteness Argument involves a commitment to RC, too.

In order for this, it is of course their first task to collect all Incompleteness Arguments there are, in order subsequently to adress them one by one. As they put it themselves, "[f]or us to be able to run [our] kind of argument we need to know [the particular single reasons] why Bach and other Moderate Contextualists think there's no such thing as these minimal propositions. What makes them incomplete? What are the criteria by which one proposition is deemed incomplete and another complete?" (C\&L 2005, 61).

In C\&L's view, the adherent of a moderate contextualism carries the burden of proof: the assumption that a sentence is semantically complete, on their account, is the standard assumption, while the view that a certain sentence is semantically incomplete requires special justification. "The challenge to the 
Moderate Contextualist", they argue, "is to show how their favorite cases of incompleteness are fundamentally different from any arbitrary English sentence" $(2005,65)$. On the other hand, the inductive structure of their argument also provides a special challenge to their own case: For, evidently, their SSA will work only insofar as they consider really each and every Incompleteness Argument, and only insofar as the SSA they give is really convincing in each case.

(4) C\&L $(2005,61)$ ask for "the criteria by which one proposition is deemed incomplete and another complete": one may think that what they thereby have in mind is a definition of "semantical incompleteness' ${ }^{19}$ This, however, is probably not what they mean. For in fact they do have and apply a sufficiently clear definition of 'semantical (in)completeness'. That a sentence is incomplete, on their account, means that it is "semantically underdeterminate" $(2005,34)$ in such a way that it "is not fully propositional [...]; it is not truth-evaluable" $(2005,7)$; "it is not the kind of thing that can take a truth value" $(2005,59) .{ }^{20}$ What C\&L probably have in mind, when they demand "criteria" of (in)completeness, is some kind of evidence for, or an indication of the semantical incompleteness of sentences.

(5) As we saw, because of the inductive structure of the (overall) SSA, its success depends on an exhaustive rejection of all Incompleteness Arguments (as well as, additionally, of all arguments in favour of context sensitivity). Now in their discussion, C\&L take exactly two Incompleteness Arguments into consideration, to which we shall turn presently. Since they end up claiming that their case is successful, they are committed to the assumption that these two arguments exhaust the range of Incompleteness Arguments. For if there were additional Incompleteness Arguments, then the inductive procedure would be incomplete, and the conclusion, that the case is successful, underdetermined.

Now let us turn to an analysis of those two Incompleteness Arguments. The first of them may be called the "Open Question test" argument. It takes the fact that a given sentence, as it stands, leaves certain questions unanswered as an indication that this sentence is semantically incomplete. Schematically, it can be stated as follows. 
The "Open Question test" argument:

(1) Some sentences are subject to Open Questions.

(2) Open Questions indicate semantical incompleteness.

Hence,

(3) There is reason to believe that some sentences are semantically incomplete.

The second Incompleteness Argument refers to a criterion which C\&L ascribe to Ken Taylor, and which produces the "felt inability to evaluate the truth value" of a sentence (the "Felt Inability", for short) as an indication of this sentence's semantical incompleteness. Schematically, it can be stated as follows.

The "Felt Inability test" argument:

(4) Some sentences trigger a Felt Inability.

(5) Felt Inabilities indicate semantical incompleteness.

Hence,

(3) There is reason to believe that some sentences are semantically incomplete.

C\&L's argument entails that each and every Incompleteness Argument commits one to endorsing $\mathrm{RC}$, and they assume that the range of Incompleteness Arguments consists of these two arguments. Accordingly, they set out to construe for either of them an SSA, intended to show that employing this Incompleteness Argument commits one to the endorsement of RC. Let us turn to an examination of the two SSAs, starting with the SSA against the Open Question test argument. 


\section{Analysis and defence of the 'Open Question test' argument}

C\&L start considering the Open Question test with reference to three groups of examples, originating from authors who, as they $(2005,62)$ suppose, are adherents of MC, such as Bach (1994b, 128ff.), Carston (1988, 167), Sperber and Wilson (1986, 188), Récanati (1993, 235), Perry $(1993,206)$ and Crimmins $(1992,17)$. The argument they develop with reference to these examples proceeds in two stages. The first stage refers to an example which Kent Bach uses in order to illustrate how the Open Question test works:

(6) "Steel isn't strong enough"

This sentence, according to Bach (1994b, 127), is "semantically or conceptually incomplete". "The conventional meaning of the sentence", he argues, "expresses not a full proposition but merely a propositional radical", which entails that the sentence, as it stands, is not truth-evaluable. Rather, "for the sentence to express a complete and determinate proposition (something capable of being true or false)", something would have to be "added". "[A] complete proposition would be expressed, a truth condition determined", by the sentence, "only if [it] were elaborated somehow" (ibid.).

Now if we assume that we must, for one or the other reason, end up with a 'semantically complete', truth-evaluable proposition, we may say that "the utterance of a semantically underdeterminate sentence requires completion", or that a certain constituent "is needed to complete the proposition" (ibid.). And to indicate just what this constituent is that "must be added for the sentence to express a complete and determinate proposition", we can refer to the fact that a certain question 'must be asked', and answered, in order to arrive at a semantically complete, truth-evaluable proposition. Thus we can say, for instance, that the sentence "Steel isn't strong enough" is semantically incomplete, and specify the way in which it is, by saying that "we need to know strong enough for what" (ibid.).

In their objection against Bach's argument, $C \& L$ introduce a counterpart to Bach's example, enriched by the words "to support the roof", which are supposed to bridge the gap diagnosed by the Open Question: 
(7) "Steel isn't strong enough to support the roof"

Although this complementation is intended to bridge the gap indicated by the Open Question, C\&L $(2005,63)$ argue, it does not achieve this task, as far as semantical incompleteness is concerned. For in fact, (7) is "equally susceptible to Incompleteness Arguments" as (6) is $(2005,62)$. It does not, for example, "settle for how long the support must last. Do a few seconds suffice? More than three days? Many days? Many years?" $(2005,63)$. Indeed, they $(2005,62)$ argue, there is no "principled difference" between (6) and (7). For "one can trigger exactly the same kind of incompleteness intuitions" for (7) as those advanced for (6) (ibid.).

Now as far as this is concerned, it is not quite certain that the adherent of MC will be particularly impressed. At least it is easy to see several ways in which she may react to this argument without conceding ground. For example, she may complain that the complement which C\&L offer, "to support the roof", is not a very promising device for the task of bridging the gap indicated by the question "Strong enough for what?": if "to support the roof" raises additional questions such as "For how long", then this only shows that it is not suitable for the task it is intended to achieve. Also, the adherent of MC may probably insist that even if such a question as "For how long?" remains unanswered, it is easy to provide another sentence, in which the remaining gap is bridged.

But now the second stage of C\&L's argument comes, and this threatens to steamroll any attempt to defend MC along these lines. It proposes that each and every sentence whatsoever is subject to one or more incompleteness-indicating questions. The idea underlying this second stage is simply to adopt an argument by Charles Travis, whom C\&L see as "an ally in this context", because "he, as a Radical Contextualist, is also in the business of showing that there's nothing special about" such a sentence as “Steel isn’t strong enough" $(2005,62)$. 
Travis' argument is intended to show that the assessment of sentences as true or false meets more obstacles than one would at first expect. Take, for instance, the sentence "The kettle is black". It may be uttered in different circumstances, including these:

Suppose that the kettle is normal aluminium, but soot covered; normal aluminium but painted; cast iron, but glowing from heat; cast iron but enamelled white on the inside; on the outside; cast iron with a lot of brown grease stains on the outside; etc. (Travis 1985, 197)

If the sentence is uttered in circumstances like these, then it appears to be impossible to say what exactly it is that 'is said', or at least impossible to say whether 'what is said' is true or false (Travis 1985, 196f.). As a consequence, if such a sentence is uttered in such circumstances, then either "it remains indeterminate precisely what was said in [these circumstances], though perhaps what was said is determinably true or false, if we only knew what it was", or else "something is said which is definitely truth valueless under the conditions depicted" (Travis 1985, 198).

The kind of questions which Travis introduces here (in the following we call them Travis-questions, and the test referring to them the Travis-question test) is similar to, although not quite identical with, the Open Question test which Bach applies. What is examined by a Travis-question is the semantic content of a sentence in a given context, and the Travis-question test confronts a given sentence with the question of whether it is true or false in the face of one or the other special context of utterance.

Travis suggests that either there is no determinate semantic content in this context, or the semantic content of the sentence in such a context is such that the sentence cannot be assessed as either true or false. Furthermore, Travis suggests that for many sentences such special contexts can be construed, contexts in which either there is no determinate semantic content, or in which the semantic content cannot be assessed as either true or false.

C\&L apply Travis's argument in order to suggest that the application of Open Question tests commits one to a radical view of semantical incompleteness. The crucial fact (or supposed fact) they refer to is that the unability to say whether the sentence is true or false in one or the other special context can be elicited, not merely with some sentences, or many sentences, but indeed with each and every possible 
sentence. On C\&L's view, those adherents of a moderate view of incompleteness who have the intuition that some sentences are truth-evaluable as they stand, while others, such as "She's ready" or "It's raining", are semantically incomplete, overlook how universally the Travis-test turns out to be applicable, given only some imaginative power. "[I]f the reason why [sentences] are incomplete is because they fail to answer these kinds of 'would it be true if ...'-questions'”, C\&L $(2005,63)$ ask, then "why should we hold that this alleged incompleteness is limited to some (more or less circumscribed subset of $[\ldots]^{21}$ sentences?"

For although, as they admit, "[y]ou might initially feel that [a sentence like "Smith weighs $80 \mathrm{~kg}$ "] requires contextual supplementation, not in the same way as 'She's ready' and 'It's raining' do", "here's a prediction: If you read a few of Travis' little stories, you will eventually have that feeling" $(2005,67)$. In fact, they contend, each and every sentence can be confronted with Travis-questions. "What Radical Contextualists see clearly, and are prepared to bravely pursue to the bitter end", they argue, "is that the same sort of incompleteness is to be found in every English sentence." (2005, 63). From the applicability of Travis-questions to all sentences (rather than merely to some, or many), C\&L conclude that if the Open Question test argument is accepted at all, then it will suggest that all sentences (rather than just some, or many) are semantically incomplete. Thus they $(2005,68)$ arrive at the view that if an argument like the Open Question test argument is applied at all, then it "also follows that RC is true".

Some of the statements with which C\&L advance Travis' argument may sound as if they actually believed that all sentences are semantically incomplete. However, they are at great pains not to subscribe to this view. ${ }^{22}$ Instead, their argument continues with an attempted reductio ad absurdum. As C\&L claim to show in their book, $\mathrm{RC}$ is quite an unacceptable view. Hence, although the Travis-test, on the assumption that it is significant, provides plausible evidence for the incompleteness of all sentences, this must not be taken as evidence that all sentences are semantically incomplete, but rather as evidence that the Open Question test must be treacherous.

Let us summarise the crucial elements of the argument schematically. The argument examines the validity of assumption (2), on which the Open Question test argument is based: 
(2) Open Questions indicate semantical incompleteness.

This assumption is challenged in the following way:

(8) All sentences are subject to Travis Questions.

(9) Travis-questions are a variety of Open Questions.

Hence, ...

(10) On the assumption that Open Questions indicate semantical incompleteness, (2) implies RC / commits one to $\mathrm{RC}$.

The conclusion represented in (10) is supposed to be devastating for a moderate view of semantical incompleteness, for the reason that the argument can be continued as follows:

(11) RC is an absurd view.

Hence, ...

(12) The assumption that Open Questions indicate semantical incompleteness implies an absurd view.

Before we turn to an assessment of the SSA, it should be noticed that C\&L's analysis of the Open Queston test argument appears to be infected by an overly liberal interpretation of the Open Question test itself. Thus, for example, some of the 'Open Questions' they construe for the sentence "John went to the gym" are not of the appropriate kind. Such questions as "Did something in the gym?", "Did what in the gym?", and "For how long?", which C\&L introduce, do not indicate any intuition of incompleteness of the sentence "John went to the gym": regardless of whether the sentence is semantically complete or not, these questions do not mark any special variety of (felt) incompleteness.

To see what went wrong, it is helpful to see exactly what C\&L $(2005,64)$ say about these questions: they argue that they can be asked - rather than that they must be asked and answered. That they can be 
asked, however, does not make them 'Open Questions' in the sense under consideration, as Kent Bach, ${ }^{23}$ rightly emphasises; for these are question which have to be answered, in order for the sentence to be truth-evaluable. Accordingly, it is quite pointless, as far as the truth-conditions of "John went to the gym" are concerned, to ask what John did in the gym. Whether he did go or not does not depend on how this question is answered. Now while those questions which can be stated are probably irrelevant, those which need to be answered are relevant for our issue. In the following I shall call questions of the relevant variety "incompleteness indicating questions" (IIQs).

Now let me present my objections to the SSA against the Open Question test. Two of my arguments are reasons not to believe premise (8), that each and every sentence is subject to one or the other Travisquestion, and the third is directed against the truth of premise (9), that Travis-questions are a variety of those Open Questions employed by some defenders of MC.

The main reason $\mathrm{C} \& \mathrm{~L}$ provide for endorsing this assumption is confidence in the pervasive applicability of Travis-questions. Against the one who assumes that there are semantically complete sentences, they $(2005,67)$ direct the contention that they are able to trigger incompleteness intuitions with respect to each and every sentence - "at least if we are allowed to talk to people about the relevant feelings"; and to the one who is still not persuaded, they make "a prediction: If you read a few of Travis' little stories, you will eventually have" the feeling of incompleteness with every sentence whatsoever $(2005,67)$.

However, considering the matter more thoroughly, some reasons emerge for the view that, even if they are allowed to talk to us, ${ }^{24}$ we should better remain skeptical. As a first reason, I should like to highlight the implausibility of what might be called an endless chain of Travis-questions and complements. If a sentence is confronted with a Travis-question, then in general there seems to be no obstacle to construing a sentence which is complemented in such a way as to avoid the question. Thus, if "Smith weighs $80 \mathrm{~kg}$ " is confronted with the question "Do we undress Smith?", then we can provide "Smith weighs $80 \mathrm{~kg}$ undressed" as a new candidate of a semantically complete sentence. 
C\&L will not hesitate to insist that for this sentence, again, some IIQ can be construed; let us take their own example: "Smith weighing $80 \mathrm{~kg}$ when?". But again a sentence can be construed which resembles "Smith weighs $80 \mathrm{~kg}$ undressed", but is complemented in such a way as to avoid the question, such as "Smith weighs $80 \mathrm{~kg}$ at 10:00 am MET, undressed". To be sure, I have no reason to claim that this sentence is semantically complete after all. The point is merely that even if there is another Travisquestion for it, again a new sentence can be construed which fills the gap indicated by it. Now when $\mathrm{C} \& \mathrm{~L}$ are claiming that each and every sentence is subject to some (apparently) IIQ, then they commit themselves to the position that this procedure, of inventing a new Travis-question, and of then subsequently construing a less incomplete sentence, can never end. But this idea does not sound very plausible after all, does it. If it is false, however, then there is no endless chain, and if there is no endless chain, then applying Open Question tests does not commit one to the endorsement of RC: the SSA against the Open Question test fails.

There is a second, and even straighter reason why the SSA against the Open Question test does not work. Consider plaitudes and absurdities, necessary truths and necessary falsities. If, for example, a sentence is necessarily false, then it is false in all contexts; thus, there can be no context in which it is not false, and hence no context in which its truth or falsity cannot be evaluated. Consider "The first human on the moon was a car manufactured by the ancient Greeks": although a speaker, in uttering the sentence, may mean one or the other thing which is true, false, or neither of both in itself, the sentence is false, whatever the context of utterance. Therefore, whatever the context, there is no IIQ for it. Similar examples are "Napoleon I., emperor of France, was a green prime number", or "Contrary to the facts, there is no difference between you and your mother". Or consider " $2+2=7$ ": since it is necessarily false, it must be false under all conditions. Therefore, however fancyful a context is construed by either Travis or C\&L, we can be sure that the sentence is false in it; thus, we can be sure that there will be no Travisquestion for it. Similar examples are "Some bachelors are unmarried", or "Some prime numbers are even numbers". 25

The third reason why the SSA against the Open Question test does not work is internal: the argument rests upon a confusion. C\&L try to convince us that each and every sentence is subject to an Open 
Question by arguing that all sentences are subject to a Travis-question. For the success of this argument it is essential that assumption (9), that Travis-questions are a variety of Open Questions, holds true. However, (9) is false. To start with, the Open Question test examines a sentence as it stands, without consideration of any particular context; while the Travis-question test concerns a sentence as confronted with a certain special context. Furthermore, if a sentence provokes an Open Question, then the incompleteness indicated is an incompleteness of its character; while in the case of a Travis-question test, the incompleteness indicated, depending on the presence of the special features of the context mentioned by the question, concerns the semantic content in such a context. Additionally, the semantical incompleteness indicated by an Open Question is categorical, while the semantical incompleteness indicated by a Travis-question is not. In the case of a Travis-question you cannot say "The sentence lacks truth conditions", full stop. When the kettle is made of aluminium and soot covered, then it may be hard, or impossible, to say whether "The kettle is black" is true. Consider, however, the case where the kettle is made of plastics and black through and through; in such a case, the availability of the Travis-question does not prevent the sentence from being true. Or consider the case where the kettle is green: in such a case, the sentence is unambiguously false. The presence of the Travis-question does not prevent the sentence from having conditions of truth and conditions of falsity; it merely shows that in addition there are conditions where the sentence's truth-value is indeterminable. ${ }^{26}$

Arguing that the pervasive availability of Travis-questions entails the universal applicability of Open Questions, C\&L apparently overlook the difference between Travis-questions and those Open Questions which, for example, Kent Bach is concerned with. Even if, as C\&L contend, each and every sentence provokes one or the other Travis-question (which, as we saw, is probably not true anyway), this does not entail that the Open Question test argument commits one to the endorsement of ubiquitous semantical incompleteness, because Travis-questions are not a variety of Open Questions. 


\section{Analysis and defence of the 'Felt Inability test' argument}

Let us turn to the second indicator of semantical incompleteness, the "Felt Inability". Taylor introduces the idea with reference to the sentence "It's raining". "[I]n the absence of a contextually provided location", he $(2001,53)$ argues, there is "a felt inability to evaluate the truth of an utterance" of this sentence. He suggests taking this as an indication that the sentence is semantically incomplete. Above, we stated the argument as follows:

The "Felt Inability test" argument:

(4) Some sentences trigger a Felt Inability.

(5) Felt Inabilities indicate semantical incompleteness.

Hence,

(3) There is reason to believe that some sentences are semantically incomplete.

Before turning to the SSA against this argument, let us deal with an additional complaint C\&L (2005, 67) produce against it: there is no reason to assume, they argue, that "psychological facts about how people feel have any bearing whatsoever on the metaphysical question of whether a proposition exists (or is complete)". This objection fails, and obviously so, because it misrepresents the "Felt Inability test" in (at least) two ways. Firstly, the conclusion aimed at in Taylor's argument is not, as they tacitly assume, a metaphysical statement, but a semantical statement. We must distinguish the claim that, given a certain possible thing (proposition), this possible thing (proposition) exists, or does not exist, from the claim that the meaning of a given sentence is, or is not, truth-evaluable. The first claim may be a matter of metaphysics, but it is not the one under consideration here. What is at issue is the second: but this is quite an innocent semantical claim (we turn back to this issue below).

Secondly, regardless of whether the Felt Inability is, or involves, a "psychological fact" or not, it is at least additionally a statement about linguistic intuition. Someone's feeling unable to evaluate the truth of a given sentence in the absence of additional information will standardly involve, among other things, her having one or the other conception of what the sentence means. If she is a (more or less) competent 
speaker of English, then it is not unreasonable to take her intuition that the sentence is not truthevaluable as (more or less strong) evidence that it is not, and to take her feeling that additional information would help as evidence that we are confronted with semantical incompleteness. Taking this into consideration, we see that C\&L's complaint is insignificant: for after all, the Felt Inabilty test argument does not, as they assume, refer to psychological facts to support metaphysical conclusions; it rather employs reference to linguistic intuitions to support linguistic conclusions.

Let us turn to C\&L's chief objection to the Felt Inability test, the SSA. ${ }^{27}$ "[I]t is possible", they $(2005,67)$ argue, "to trigger [a Felt Inability] with respect to any sentence whatsoever". In order to convince us, they employ two examples, confronting each of them with several Travis-questions. The first example is "Smith weighs $80 \mathrm{~kg}$ ". Although the meaning of this sentence may at first appear to be truth-evaluable, they argue, this appearance can be unsettled by means of questions such as "Smith weighing $80 \mathrm{~kg}$ when?", "Under what conditions are the weighings done?", "Do we undress Smith?" $(2005,67)$. The same holds for the sentence "That's a red apple", considering questions such as "Red where?”, “In what light?”, “Scrubbed?”, “Brushed?", “Painted?” (2005, 68). In the face of cases like these, C\&L conclude that "there's no reason to think [that Felt Inabilities] are triggered with respect to just a limited subset of English sentences" $(2005,68)$. Rather, "it is possible to trigger these feelings with respect to any sentence whatsoever, at least if we are allowed to talk to people about the relevant feelings", they $(2005,67)$ contend.

As in the case of the Open Question test, the clue of the SSA is the (allegedly) ubiquitous applicability of Travis-questions; in the present case this is connected with the assumption that Travisquestions trigger a Felt Inabilty; the assumption that Felt Inabilities indicate semantical incompleteness thus appears to lead to RC. More precisely, the structure of the argument is this.

(13) On assumption (5) of the Felt Inability test argument, that a Felt Inability indicates the semantical incompleteness of the sentence considered, a moderate view concerning the extension of semantical incompleteness is indicated if some but not all sentences trigger a Felt Inability, while a radical view is indicated if all sentences do so. 
(14) Each and every sentence allows for Travis-questions, and Travis-questions always trigger a Felt Inability.

Hence, ...

(15) On assumption (5) of the Felt Inability test argument, a radical view of the extension of semantical incompleteness is indicated.

(The argument proceeds as in the case of the Open Question test argument.)

The reasons why this SSA does not work resemble those introduced in the case of the Open Question test argument - which is not surprising if we take into consideration that again the clue of the argument is the allegedly ubiquitous applicability of Travis-questions. Thus, the first reason why the SSA fails is the implausibility of an endless chain of Travis-questions and complements. Assuming that the defender of MC reacts to each Travis-question by construing a sentence which is less incomplete, and the defender of VMC continues each time trying to invent a new Travis-question, it is not very plausible to assume that the chain will continue strictly ad infinitum. But if it does not, then this suggests that premise (14) is not true, which leads to the breakdown of the argument.

The second way in which the SSA fails was already mentioned with the Open Question test argument, too. There are sentences which appear to be truth-evaluable in each and every context, just because their truth or falsity holds trivially, or necessarily. This is good reason to believe that C\&L, even if they are allowed to talk to us, will be unable to construe any Travis-question for them. But if there really are such sentences, which are not subject to any Travis-questions, then there is a second reason why the SSA against the Felt Inability test argument does not work.

There is a third reason. The SSA rests on the assumption that Travis-tests trigger a Felt Inability, which is used to suggest that using the Felt Inability as an indication of semantical incompleteness leads to the conclusion that all sentences are semantically incomplete. However, this assumption is false. Although it may be that Travis-tests trigger a felt inability to evaluate the truth of a sentence, what they 
trigger is not a Felt Inability of the kind under consideration here. (For obvious reasons, the argument resembles the third argument in the case of the Open Question test argument.)

Firstly, the Felt Inability which the defender of MC is supposed to be concerned with is triggered by considering a sentence as it stands, without considering one or the other special context. The feeling triggered by Travis-questions, in contrast, is triggered by a sentence as applied to one or the other special context. Secondly, the feeling triggered by a Travis-question indicates semantical incompleteness of the semantic content of the sentence in a particular context; the Felt Inability referred to by the defender of MC, however, is supposed to indicate the semantical incompleteness of the character of the sentence under consideration, without consideration of any particular context. Thirdly, the Felt Inability which the defender of MC is concerned with indicates categorical incompleteness; the Travis-test, however, does not; for a sentence may lack truth-conditions under the special circumstances brought in by the Travis-question, while still having truth conditions in other contexts. Thus, taking C\&L's example, the felt inability to evaluate the truth of "That's a red apple", as confronted with the question "Red where?", does not indicate that the sentence has no truth conditions at all, full stop; it merely indicates that it lacks them in case the apple is red somewhere, but not everywhere. The felt inability triggered by the question does, for instance, not tell against the view that if the apple is red through and through, then the sentence is true, or that if the apple is green with brown spots, the sentence is false.

Thus, after all the SSA against the Felt Inability test argument fails for at least two reasons. Firstly, the assumption it makes, that every sentence is subject to Travis-questions, is false. Secondly, the felt inability triggered by Travis-questions is not of the type under consideration in the Felt Inability test argument, such that even if every sentence were subject to Travis-questions, there would be no danger that RC follows from the application fo the Felt Inability test argument.

So the SSA against Incompleteness Arguments fails, because the SSAs against both Incompleteness Arguments do not work, for more than one reason in either case. Over and above this, however, there is another reason why the SSA against Incompleteness Arguments fails. As was emphasised above, the 
(overall) SSA depends in its soundness on the assumption that the two Incompleteness Arguments which C\&L take into consideration, the Open Question test argument and the Felt Inabilty test argument, really exhaust the range of Incompleteness Arguments. However, this is not the case: additional Incompleteness Arguments - additional arguments in favour of semantical incompleteness - can be construed.

We may, for example, take the impossibility to evaluate a sentence's truth (rather than the feeling that evaluating it is impossible, as in the case of the Felt Inability test) to be an indication of its semantical incompleteness, given that, in addition, the sentence does not support the derivation of any trivial falsities. This test might be built on the assumption that there are exactly two occasions for non-truthevaluability, semantical incompleteness and self-contradictoriness. To be sure, for my argument it is not important that this test is quite appropriate; it would fully suffice that it, or something like it, can be used to build another Incompleteness Argument.

Another possible indicator of semantical incompleteness (deriving from an incompleteness of character), and one which C\&L actually may be expected to appreciate indeed, is a sentence's containing one or more indexical expressions. Thus one may assume that while "She's happy" is semantically incomplete, "Napoleon I. Buonaparte is happy" is semantically complete, or at least semantically incomplete to a lesser extent. Yet a third possible indicator of semantical incompleteness neglected by $C \& L$ is the presence of vague expressions (in the sense mentioned by C\&L $(2005,145)$. Thus, for whatever deeper reason, one may suppose that compared with "Peter is huge", "Peter is 1,80 $\mathrm{m}$ tall" is either not semantically incomplete at all, or at least less incomplete.

These three hints are, of course, not meant to give any systematic account of Incompleteness Arguments, or indicators of semantical incompleteness. They do suffice, however, to show that the Open Question test argument and the Felt Inability test argument can not be passed off as the only Incompleteness Arguments. The range of possible arguments in favour of a moderate view concerning semantical incompleteness is less poor than C\&L believe. Given this, and given the inductive structure 
of the SSA, however, there is another way in which the SSA against incompleteness Arguments turns out to be inconclusive.

What is more, pursuing the issue further on, things turn out to be even worse for C\&L's argument. Consider what happens when $\mathrm{C} \& \mathrm{~L}$ try to strengthen their inductive reasoning by really taking all Incompleteness Arguments into account. Let us, as an example, consider the second case just mentioned, where the presence of indexical expressions is taken as an indication of semantical incompleteness. In order to avoid the breakdown of their SSA, C\&L have to construe an argument to the effect that if we take indexical expressions as an indication of semantical incopleteness, then RC concerning semantical incompleteness follows. Furthermore, there is a Context Shifting Argument to the effect that sentences containing indexicals are context sensitive, too, and thus C\&L will additionally be forced to construe and subscribe to an argument to the effect that such a view leads to radicalism concerning context sensitivity. However, in fact they cannot consistently subscribe to arguments like these. For in fact their own theory assumes the presence of semantical incompleteness and context sensitivity in cases involving indexicals. Following the rationale of their objection, this would commit them to giving some argument in favour of context sensitivity and semantical incompleteness in the case of indexical expressions. Now if such arguments did commit one to RC, then C\&L would, following their own SSA, be comitted to the endorsement of $\mathrm{RC}$, and insofar it is true that $\mathrm{RC}$ is quite unacceptable, as they continue arguing, they would commit themselves to quite an unacceptable view. In short, the attempt to save their SSA by completing the inductive argument would force them to disprove their own theory by this very SSA.

The general problem underlying this dilemma has to do with the structure of C\&L's own account, which suffers from an ambiguity between contextualism and non-contextualism. While their SSA against MC pretends that their views are decidedly anti-contextualist, and although they call their view "semantic minimalism" and present it as an (or rather, the) alternative to "contextualist" accounts, their own theory does contain several contextualist elements, too. Thus, as we saw, they do assume context sensitivity and/or semantical incompleteness in the case of indexical expressions, vague expressions, and imprecise expressions. Given this ambiguous structure of their account, the SSA cannot be showing, 
as it is intended to, that all contextualist accounts fails while C\&L's account lives: any argument which really provides an exhaustive rejection of all contextualist elements will additionally be disproving C\&L's own account, too.

\section{Are Incompleteness Arguments metaphysical arguments?}

Before turning to C\&L's third main argument, let us briefly consider a remark which they make quite much in passing, and which may appear as critically directed against Incompleteness Arguments, too, but which is in no way worked out, and which in any case can be dismissed rather easily. Both the Open Question test and the Felt Inability test appear to involve intuition, though in slightly different ways. Now in the course of the discussion, $\operatorname{C} \& L(2005,61)$ appear to suggest that the reference to "brute intuitions" is in one or the other way illegitimate. They ask their opponents for an argument in favour of MC, "beyond just appeal to brute intuitions" (my emphasis). They do not, however, make any suggestion what the problem with the reference to intuition might be. And in fact they cannot be expected to have any general concern about it, for they do employ reference to intuitions for their own argumentative purposes in other places. ${ }^{28}$ Thus, we can assume that $\mathrm{C} \& \mathrm{~L}$ have no general reservations against arguments in terms of intuition, after all.

But let us now turn to C\&L's third argument against Incompleteness Arguments. Over and above their SSAs, they try to rebut Incompleteness Arguments with reference to the (alleged) fact that they are metaphysical arguments. "Moderate and Radical Contextualists who use such arguments", they (2005, 11) conjecture, "are typically deeply confused about the relationship between semantics and metaphysics". "These arguments are not about language", they contend, but "they are about various non-linguistic aspects of the world"-thus, instead of being semantical arguments, they are metaphysical arguments, they argue.

This diagnosis may strike us as a bit surprising. They establish it as follows. Referring to an argument we dealt with above, they $(2005,61)$ start by saying that "[a]ccording to Bach, [the sentence 
"Steel isn't strong enough"] doesn't express genuine truth conditions". This is indeed the claim we know from Bach's $(1994 b, 127)$ article, and at least at first sight it does not appear very much as a metaphysical claim. But C\&L continue by giving three reformulations of the claim. The first reformulation is "There's no proposition of the sort that steel isn't strong enough". This already sounds a bit like a metaphysical claim. The second reformulation is "The italicized expression in the previous sentence just doesn't refer to something truth-evaluable". The third reformulation of Bach's argument is "There's no such thing as steel not being strong enough simpliciter". Now this third reformulation, indeed, heavily sounds like a metaphysical claim, namely, a statement about the question whether a certain kind of property (being strong enough simpliciter) exists or not. Consider also the following description they give of Incompleteness Arguments:

Incompleteness Arguments are always simple (so simple that they might not deserve the moniker 'argument'). Typically, all they amount to is a claim like the following:

Consider the alleged proposition that P that some sentence S semantically expresses. Intuitively, the world can't just be $\mathrm{P}$ simpliciter. The world is neither P nor not P. There's no such thing as P's being the case simpliciter. And so, there is no such proposition. (C\&L 2005, 11)

In this passage, the Incompleteness Argument appears as a statement concerning the question whether a proposition such as 'that $\mathrm{P}$ is the case' (simpliciter) exists. Again, this sounds like a metaphysical statement (namely, concerning the existence of certain propositions). ${ }^{29}$

Now the first question we may ask ourselves is, even if the Incompleteness Argument really were a metaphysical argument, why should this be considered as problematic? According to C\&L, the reason is twofold. Firstly, Incompleteness Arguments would turn out to be inconclusive; for "even if they were good arguments, $[\ldots]$ no semantic conclusions [would] follow from these arguments even if they were sound". Secondly, C\&L claim that "considered [...] as metaphysical arguments, they are unsound" $(2005,11)$

Apparently, C\&L assume that if an argument is a metaphysical argument then it cannot possibly be used to support semantical conclusions. The first problem with their argument is then, however, that this assumption actually is not very plausible. We can, for instance, use a meteorological premise, such as 
the assumption that tomorrow there will be a hurricane in Barcelone, in order to support a football conclusion, such as that Barça will fail to get in the lead in the Primera División (because their match will have to be cancelled). Indeed, it also appears that we can, for example, use the metaphysical premise that there can be no non-descriptive meaning to support the semantical conclusion that the meaning of interrogative sentences cannot be non-descriptive. Thus, while I cannot see any reason why we should endorse the premiss C\&L seem to employ, that metaphysical arguments cannot support semantical conclusions, we have reason to avoid this assumption. If we avoid it, however, then C\&L's argument appears to break down even before starting.

Anyway, the argument fails for a second reason. Let me begin with a comment on the paraphrases C\&L provide of Bach's argument. If I state, about the coffee on my desk, that it is not very hot, then this may be paraphrased approximately as the claim, about a possible very hot coffee standing on my desk, that it does not exist, and about a colder one standing on my desk, that it does exist. Stated this way, my complaint may appear as a metaphysical claim. Probably, it would be possible to paraphrase virtually every statement in a similar way, replacing ascriptions of property with metaphysically-sounding double ascriptions of existence. However, this does certainly not justify the conclusion that the original claim is a metaphysical one. My remark about coffee, for example, is rather not (as its author I am in a good position to tell). If C\&L's test says otherwise, then this is an indication that the test is not reliable.

Keeping this in mind, let us turn to C\&L's favourite example. Kent Bach (1994a, 269) says about sentences such as "Steel isn't strong enough" that "though syntactically well formed, [they] are semantically or conceptually incomplete, in the sense that something must be added to the sentence for it to express a complete and determinate proposition". As we saw, C\&L provide several paraphrases of this argument, such as “There's no proposition of the sort that steel isn't strong enough", and "There's no such thing as steel being not strong enough simpliciter", which sound like metaphysical statements (concerning the existence of propositions in the one case, and of facts in the other). These paraphrases, however, are indeed misleading. What Bach intends to express with his words is not a statement about certain propositions, to the effect that they do not exist. Neither is it a statement about certain possible facts, to the effect that they do not exist. What he aims at, rather, is a statement about certain meanings, 
to the effect that they are not truth-evaluable, because as they stand they are somehow incomplete. In particular, Bach does not, as one of C\&L's paraphrases insinuates, deal with the question whether 'that steel isn't strong enough' exists or not. Instead, he is concerned with the quite different question whether 'that steel isn't strong enough' is truth-evaluable or not. Stated in an unpretentious way, this question does not even sound like a metaphysical one. It is reasonable enough, I think, to assume that this defence applies to other Incompleteness Arguments as well. C\&L's argument fails, because the surprising premise it relies on is unsound: Incompleteness Arguments are by no means metaphysical arguments.

\section{Summary}

Let me summarise the findings of this paper. We were examining the central argument of $I S$, an argument 'by elimination', which says that there is no acceptable alternative to C\&L's very moderate contextualism. They try to convince us of this view by assuming that there are exactly two alternatives to their account, MC and RC, and by arguing that RC is inconsistent and absurd, while MC collapses into RC. The alleged reason for this collapse is that each and every argument in favour of either context sensitivity or semantical incompleteness commits one to the endorsement of $\mathrm{RC}$, because for each such argument a (partial) SSA can be construed, showing that the application of this argument cannot be restricted to any finite number of sentences, but will extend to literally each and every sentence. Additionally, they suggest that Incompleteness Arguments use metaphysical assumptions for conclusions about language, which on their view makes them.

As our discussion showed, however, the arguments they give are not convincing. First of all, it is not true that $\mathrm{MC}$ and $\mathrm{RC}$ are the only alternatives to their account. The assumption on which this view apparently rests, that context sensitivity and semantical incompleteness are either the same thing or at least coextensional, is unfounded. Building on the assumption that the identity of context sensitivity and 
semantical incompleteness can not just be taken for granted, I suggested adopting the view that there is no context sensitivity ("Real SM", as I call it), together with a moderate view of the extension of semantical incompleteness. Among the advantages this view has is the fact that by completely rejecting the context sensitivity of semantic content it is more obviously consistent with the Basic Idea of C\&L's account than their own theory. Assuming this account, the SSAs against context sensitivity can be ignored, while the SSAs against Incompleteness Arguments remain significant.

These, however, turn out to be unconvincing, for several reasons. C\&L take two kinds of Incompleteness Arguments into consideration, Open Question test arguments and Felt inabilty test arguments. The success of their SSA depends on the assumption that the two arguments exhaust the range of possible Incompleteness Arguments. They really do not, however, and this is one reason why the SSA against Incompleteness Arguments fails. Furthermore, the SSAs which C\&L direct against those two Incompleteness Arguments are unsuccessful, too. It is possible to employ Open Questions and Felt Inabilities as indications of semantical incompleteness without being commited that all sentences are semantically incomplete, because neither do Travis-questions indicate the relevant kind of semantical incompleteness, nor is it the case that all questions provoke Travis-questions. It does not seem that they are able to keep their promise, to construe Travis-questions for each and every sentence.

Finally, Incompleteness Arguments do not fail by virtue of being metaphysical arguments for a semantical conclusion. First of all, they appear to assume that metaphysical arguments cannot support semantical conclusions; but they do not explain why we should believe such a thing. Furthermore, the interpretation of Incompleteness Arguments as metaphysical claims, which they assume, rests on several misleading formulations they give of these arguments. Insofar as these formulations do present Incompleteness Arguments as metaphysical statements, they misrepresent what the defenders of Incompleteness Arguments have in mind; thus, there is no reason why the adherent of Incompleteness Arguments should subscribe to these inappropriate 'versions' of 'her' claim.

So the central aim of $I S$, the attempt to prove the truth of their own account by eliminating all other accounts, turns out to collapse for quite a number of reasons. Their view of the range of competing 
accounts is too narrow, ${ }^{30}$ the structure of their argument is inconclusive, and the premisses on which their argument rests are unsound. Thus the claim they make, that their own theory remains as the only game in town at the end of the day, turns out to be too optimistic after all.

\section{(i) Anmerkungen}

${ }^{1}$ I should like to thank $\square$, Ernie Lepore and $\square$ for their valuable hints and comments on this and an earlier draft. Work on the issues dealt with in this paper was supported by

2 Cappelen and Lepore $(1997,278)$ characterise 'content' as "whatever semantic theory attempts to elucidate". This suggests that on their account 'semantic content' refers to a variety of the semantic meaning of (declarative) sentences-Kaplan's (1989) "character", for example, being another variety. That their 'semantic content' is a kind of semantic meaning is reconfirmed by the 'Basic Idea' which is just being introduced: for although it is plausible to maintain, about semantic meaning, that it is what someone ignorant about the characteristics of the context can know, it is hard to see why such a thing should be maintained of 'semantic content' if this does not refer to a variety of semantic meaning. An alternative interpretation of their account is given, for example, by Korta \& Perry (2007, 95f.). According to it, 'semantic content' is the result of the application of pragmatic impact on semantic content, which suggests that 'semantic content' is a matter of pragmatics; if we ascribe this notion to C\&L themselves, however, then their statement of the Basic Idea would be hard to explain.

${ }^{3}$ To say that the context has no significance for the semantic meaning of an expression is, of course, not to deny that there may be a contribution of the context to what the speaker means with the expression, or what the speaker means in making the utterance, etc.

4 See, e.g., 2005, 6, n5, 128, 131.

${ }^{5}$ See, e.g., 2005, 6, 14, 31.

6 They give different characterisations in both respects; see, e.g., 2005, 6, 14, 31, 128, 131; cf. also 6, n5.

7 They sometimes ascribe additional claims to MC and RC; see, e.g., 2005, 6f.

${ }^{8}$ For details see C\&L 2005, Chapter 7.

${ }^{9}$ For details see C\&L 2005, Chapter 8.

${ }^{10}$ For details see C\&L 2005, Chapter 9.

11 They $(2005,10)$ call this a "methodological observation"; see also C\&L 2005, 10, 14, 38.

12 To be sure, it is not important for the present purpose that non-coextensionality is, or can be proven; the crucial thing is that coextensionality cannot be taken for granted.

13 This minimal view of context sensitivity is similar, e.g., to Robyn Carston's (2008) form of 'minimalism' and to Korta \& Perry's $(2007,99)$ 'KP minimalism'; cf. also Borg’s (2007) conception of 'minimalism'.

14 See, e.g., C\&L (2005, 2-3). 
15 C\&L give different descriptions of how far, on their account, semantical incompleteness and context sensitivity are supposed to extend. Initially $(2004,1)$ they suggest that context sensitivity is restricted to the determination of the semantic value of 'indexicals' in Kaplan's sense, but then they weaken the restriction by the qualification "plus or minus a bit" (2004, 2); and they later (2004, 144f.) include, additionally, disambiguation and precisification.

${ }^{16}$ See C\&L (2005, 11, 33-35, 59-61).

17 See also C\&L 2005, 14, 60, 61.

18 This interpretation is also suggested by their view, already mentioned above, that as a consequence of $\mathrm{SSA}, \mathrm{RC}$ is the negation of $\mathrm{VMC}$.

${ }^{19}$ Cf. also (2005, $\left.62 \mathrm{n} 1\right)$, where C\&L deplore the lack of "any sharp definition of 'in/complete' in [the] literature".

${ }^{20}$ For sufficiently clear conceptions of the notion of semantical incompleteness see also, e.g., Bach 1994, 126f., or Sperber and Wilson 1986, 188.

21 "English", they qualify here; however, being about meaning in general their argument should not be restricted to English sentences and meanings.

22 Cf., e.g., C\&L $(2005,59)$.

${ }^{23} I d ., 2006,441$.

${ }^{24} \mathrm{I}$ did ask them to talk to me: I gave them the examples used in the following, among others, and requested them to give arguments why they think they are semantically incomplete, giving an Open Question for them, and eliciting in me a Felt Inability concerning them. Ernie Lepore said it was too long ago, and I should better ask Cappelen. Cappelen did not deal with the actual task at all, even after I repeated my request.

25 Again, what a speaker means in uttering those sentences may be true or false or neither: but what the speaker means is to be distinguished from what the sentence means.

26 The situation in the case of Travis-questions, as truth conditions are concerned, appears to be quite much the same as with the traditional analysis of "The present king of France is bald": if there is no king of France, then we have failure of presupposition and some such problem as lack of truth value; while if there is a king of France, then this problem disappears.

27 As with the Open Question test argument, the argument goes back to thoughts by Charles Travis (see, e.g., id. 1996); Travis himself, however, does not state the argument as explicitly as C\&L do.

${ }^{28}$ See, e.g., C\&L 2005, 1, 62 n1, 151.

${ }_{29}$ The sketch C\&L draw here of Incompleteness Arguments is inconsistent concerning the question what kind of metaphysical issues Incompleteness Arguments are supposed to answer: either the existence of certain propositions, or else the existence of certain properties.

30 There are additional types of views which their account either ignores or overlooks; see 


\section{References}

Bach, K. (1994a). Semantic Slack: What is Said and More. (In S. Tsohatzidis (Ed.), Foundations of Speech Act Theory (pp. 267-291). London: Routledge.)

Bach, K. (1994b). Conversational Impliciture. Mind \& Language, 9/2, 124-162

Bach, K. (2006). The Excluded Middle: Semantic Minimalism without Minimal Propositions. Philosophy and Phenomenological Research, 73/2, 425-442

Borg, E. (2007). Minimalism versus Contextualism in Semantics. (In G. Preyer \& G. Peter (Eds.), Context-Sensitivity and Semantic Minimalism (pp. 339-359). Oxford: Oxford University Press.)

Cappelen, H. \& Lepore, E. (1997). On an Alleged Connection between Indirect Speech and the Theory of Meaning. Mind \& Language, 12/3-4, 278-296

Cappelen, H. \& Lepore, E. (2005). Insensitive Semantics: A Defence of Semantic Minimalism and Speech Act Pluralism. (Oxford: Blackwell)

Carston, R. (1988). Implicature, Explicature, and Truth-Theoretic Semantics. (In R. Kempson (Ed.), Mental Representation: The Interface between Language and Reality (pp. 155-181). Cambridge: Cambridge University Press.)

Carston, R. (2008). Linguistic Communication and the Semantics/Pragmatics Distinction. Synthese, $165,321-345$

Crimmins, M. (1992). Talk about Beliefs. (Cambridge, MA: MIT Press)

Kaplan, D. (1989). Demonstratives. (In J. Almog et al. (Eds.), Themes from Kaplan (pp. 481-563), Oxford: Oxford University Press.)

Korta, K. \& Perry, J. (2007). Radical Minimalism, Moderate Contextualism. (In G. Preyer \& G. Peter (Eds.), Context-Sensitivity and Semantic Minimalism (pp. 94-111). Oxford: Oxford University Press.) 
Perry, J. (1993). Thought without Representation. (In id., The Problem of the Essential Indexical and Other Essays (pp. 205-225). Oxford: Oxford University Press.)

Récanati, F. (1993). Direct Reference: From Language to Thought. (Oxford: Blackwell)

Sperber, D. \& Wilson, D. (1986). Relevance. (Oxford: Blackwell)

Taylor, K. (2001). Sex, Breakfast, and Descriptus Interruptus. Synthese, 128/1-2, 45-61

Travis, C. (1985). On What is Strictly Speaking True. Canadian Journal of Philosophy, 15/2, 187-229 